\title{
UNDERSTANDING, SUPERVISION, STRATEGY AND ACCEPTANCE EFFECT INTO THE BLOCKCHAIN EMPLOYMENT IN MALAYSIA
}

\author{
MAHADI HASAN MIRAZ ${ }^{1}$, MOHAMMAD TARIQ HASAN ${ }^{2}$, FARHANA RAHMAN SUMI ${ }^{3}$, \\ SHUMI SARKAR $^{4} \&$ MOHAMMAD ISMAIL MAJUMDER ${ }^{5}$ \\ ${ }^{1}$ School of Technology Management and Logistics, Fellow, Universiti Utara Malaysia (UUM), Malaysia \\ ${ }^{2}$ Assistant Professor, School of Business and Economics, United International University (UIU), Bangladesh \\ ${ }^{3,4}$ Assistant Professor, Department of Business Studies, University of Information Technology and Sciences (UITS), Bangladesh \\ ${ }^{5}$ Bangladesh University of Professionals, Bangladesh
}

\begin{abstract}
Throughout history, the food industry has been responsible for several crises and, as the market becomes more global, it is difficult to keep information under control and confidence. As a result of food scandals, asymmetry in information increases customer awareness, as well as initiatives such as evidence to address problems. To increase transparency, the information infrastructure is continuously evolving and, more recently, blockchain technology has paid great attention to possible solutions to issues. Blockchain is an open, distributed and decentralized authentication system for digital transactions, where transaction data is stored securely in the so-called blocks that make up a network. In order to obtain effective food retail, it is necessary to monitor specific product information in the food retail industry.The study concludes that blockchain technology is still immature for food retail, and the biggest challenge is to create a culture that promotes collaboration, exchange of information and easy-to-accept standardization. However, the blockchain technology allows safe and transparent tracking functions, with traceability and a framework that can save costs and the environment when removing a product. In addition, to be willing to cooperate and make time and effort in new implementations, it is essential to find the real value of the application for all interested parties.
\end{abstract}

KEYWORDS: Blockchain, Food Retail Market \& Blockchain Implementation in the Retail Food Market

Received: Jun 06, 2020; Accepted: Jun 26, 2020; Published: Aug 18, 2020; Paper Id.: IJMPERDJUN2020793

\section{INTRODUCTION}

\subsection{Outline of the Research}

Overall this thesis consists of an introduced background of blockchain in the retail market. The discussion leads to the construction of the problem statement and description of the objectives that need to be achieved in this research.

\subsection{Retail Industry in Malaysia}

The retail industryis a vital role in Malaysian for several motives and its impact on the local market (Koho, Tapaninaho, Heilala\& Torvinen, 2015). In Asia, Malaysia is one of the biggest exporters of manufacture (Garetti\&Taisch, 2012). In addition, the retailing process of the manufacturing industries in most famous for developing retail marketing. In addition, retailing is one of the largest in Malaysia and is still expanding in urban and countryside. However, this faces significant challenges because the nation does not produce enough raw materials, unfavourable trade policy and inadequate spread opportunities. Consequently, Malaysia’s retail industries did not expend and did not adapted the current finance technology (FinTech) in the retail market. 
Furthermore, the packaging industry is one of the most dynamic manufacturing industries in the world for retail trade, environmental change, market volatility, volatile customer demand, complex business dynamics, short life cycles and entry barriers. In addition, retail sectors have developed distribution networks, technology transfer and public cooperation. Globalization and globalization have evolved rapidly (Tognetti, Grosse-Ruyken and Wagner, 2015). The association of manufacturing retailers in Malaysia and the production of individuals should improve the quality of their products. Cryptographic currency can only be achieved by addressing challenges in specific areas such as infrastructure, compliance, labour supply, supplier performance, raw materials, political stability (Brennan and Lunn, 2016, Clancy, 2016, CNBC, 2015).

\subsubsection{Retail Industry \& Its Influence}

The common characteristic of the retail trade is the dynamism of the clients and races exist in different levels of distribution structure(Mahadi Hasan Miraz, Saleheen, Habib, \& Ramli, 2012). In Malaysia, the retail sector has undergone rapid changes since the early 1990s with new retail concepts that compete with more traditional forms of retail. If Malaysia's retail outlets dominate in the 70s and 80s and local shops are dominated, there are now hypermarkets and 24hour stores(Saleheen, Miraz, Habib, \& Hanafi, 2014). The range of products and customer comfort are some of the key elements that attract Malaysian customers to new points of sal(Weldon, Herridge, Cohen, \& Technology Solutions, 2017).

This new scenario involves the consequences of increased competition(Bansal \& Bansal, 2018). Existing retailers represent a potential threat to the emergence of new and larger retailers, especially if the majority of the wholesalers are multinational corporations with local partners (Jonker, 2018). When one considers that size and financial capacity are potent weapons that kill inferior rivals, large retailers are often considered a member of the dominant channel capable of eliminating smaller rivals (Saleheen et al., 2014). At the same time, the company agreed with the fact that to a certain extent, and larger retailers can launder less efficient retailers(Weldon et al., 2017). However, balanced and fairer regulation is also needed to ensure that retail development more importantly. This research assesses the potential elements that affect the ability of retailers to compete effectively with retailers prospects throughout the entire Malaysia medium retail industries (Weldon et al., 2017).

\subsection{Supply Chain Management}

Supply Chain Management (SCM) defines sales, upstream, and downstream customer associations as the best value for the customer (Dubey \& Ali, 2013; Parkhi et al., 2015). Also, SCM is an idea that accomplishes the flow of factual, data and money to the descending members from upwards to downwards. It similarly arrangements with the placement of the factual subsequently ingesting in accordance with environmental standards. Furthermore, SCM seeks to accomplish this efficiency at the lowest possible price (Dubey et al., 2012). The management of the supply chain involves the management of supply and demand within companies (Randall \& Mello, 2012). Moreover, SCM makes the process of improving business processes more flexible, agile and more competitive. In spite of main task of SCM is to progress the competitiveness of a package (Machowiak, 2012). Moreover, Supply Chain Management is the incorporation of different ideas, such as the expansion of the company, the virtual organization, the computer-generated chain and the new hybrid supply chain. As well as considerations are important for the strategy and operation of the industry (Walton \& Gupta, 1999). Altogether, SCM is responsible for recognizing and applying approaches that diminish costs and maximize reliability, progressively modest and multifaceted markets (Wadhwa et al., 2008). Together with the SCM is integrated 
with blockchain for better performance and effective for the industries and retail market.

\subsection{Blockchain}

A distributed and decentralized catalogue that is used to maintain a constantly increasing list of histories is called blockchain. Every blockchain covers a timestamp and a connection to an earlier block (Kakavand, Nicolette and Bart, 2016). The design and destination block networks are inherently resistant to data modification. Functionally, the block network can serve as open and distributed accounting that can capture transactions between the two parties in an efficient, verifiable and permanent manner (Bruya, 2017; Blockchai wiki, 2018). The block is where the block switch saves our data. The block network saves the cluster data at the same time, instead of streaming and stored at the same time can be called a blockchain. The blocks are organized as a link so the name "blockchain".

In this section, a detailed background blockchain is required and the parties complicated. The blockchain originates from its mechanical building of a Block Chain (Schatsky \& Muraskin, 2015). Every block is connected to the preceding block by a cryptographic hash. The block is a data structure that holds premises lists. The transactions are made by peer to peer by through blocks and the modification of the block network. As such transactions can exchange money, but it is not confined solely to financial transactions by permitting within the arbitrary code.

Furthermore, get specific differences between blockchain permission and without permission of blockchain as well as describe the different participants of these blockchain networks (Know et al.,2014). According to the seabed, this sea can be used as a system, which is located on the bottom of the sea. Each Candidate Block Matches the Participant Who agrees to protocol consensus in the congregation. As such, anyone can be accumulating communications inside a block and add this block to the blockchain (Beikverdi\&JooSeok, 2015). Related works are also known as writer validators, which will no longer require permission reduction but in the process of creating transactions, merely reading, analyzing and auditing to blockchain.

\subsection{Properties of Blockchain}

In this section, we designate and liken the utmost important features of distributed ledgers and centralized systems which are known as the blockchain.

\subsubsection{Public Verifiability}

Public audibility allows anyone to check the accuracy of the system status. In a distributed accounting ratio for each transition are strengthened (eg Blockchain miners), which can be a limited number of participants (Christidis, 2016). However, any observer can check whether the general ledger's status has changed according to the protocol. In addition, every observer finally receives the same view recorded for at least a certain length. In a centralized system, different observers can get a completely different picture of the system. As such, we may not be able to check if all system tran sition has been correctly implemented (Huang. Et.al, 2016). Instead, users have to trust the central unit to be in the right position and transaction among clients.

\subsubsection{Transparency}

The transparency of the data is the process of updating the state as a requirement for system control (Huh. Et.al, 2017). However, the amount of transparent information for each participant must have access to all information. 


\subsubsection{Privacy}

Privacy is an inherent part of every system. Avoid getting into the system and enjoying it without interruption by other online malware. Data protection is a more easy fundamental system in a centralized network because system control changes are not necessary for system operation (Armknecht, Et.al, 2015).

\subsubsection{Integrity}

The integrity of the information guarantees that information is protected from modifications, permissions and can be corrected for the information contained therein. In addition, it is work through a verifiable proportional system with unique control of the integrity of the data-furthermore, the contrast and the integrated system guaranteed for the central system (Vasek, 2015).

\subsubsection{Redundancy}

Data redundancy is essential for several uses. In blockchain systems, redundancy is initially provided through writer's replication. In central systems, redundancy is usually achieved through replication through other physical servers and backups (Sing. Et.al, 2016).

\subsubsection{Trust Anchor}

The trust anchor determines who represents the highest level of authority in a system that is entitled to grant and not allow read and write access to a system (Kaskaloglu, 2014).

\subsection{Where Does a Blockchain Make Sense}

In general, the use of an open or authorized Blockchain is only appropriate if many mutually mistrustful individuals wish to communicate and are not prepared to rely on a trustworthy third party online. We find one or more parties who write the system property, i.e. a writer corresponds to a written entitute in a standard database system or a blockchain participant (Aitzhan, Davor 2016). If no data needs to be processed, a collective database is not requested but blockchain. Likewise, each blockchain writer provides additional guarantees and a standard database that offers better output for efficiency and latency. In addition, there are two options for the trusted third party (TTP) transaction. First, if the TTP is still online, writing can be delegated and will serve as a verifier for accurate transitions. Second, if the TTP is normally offline, it may serve as a certificate authority for blockchain permissions, i.e. where all device writers are identified. If the authors trust each other they all assume that no participant is malicious; the best solution is likely to be a database with shared written access (Ziegeldorf. Et Al, 2016). If they don't trust each other, it makes sense to use a blockchain permission. Depending on the criteria for public verification, anyone may be able to read system properties (public blockchain permissions) or the collection of readers may be limited (private blockchain permissions). If there is no fixed collection of writers and the participants know about them, blockchain is the best option for digital transactions (Heilman. Et.al, 2016).

We analyze different articles and discover certain permission-less properties and blockchains in a central database. In a centralized network, latency and efficiency in blockchain systems is usually much higher (Natoli and Gramoli, 2016). However, through their consensus process, blockchains add additional complexity. For example, Blockchain currently has about seven transactions per second which could be extended to about 66 without sacrificing safety. A centralized system like Visa, on the other hand, can accommodate peaks of more than 50,000 transactions (Shi, 
2016). A large number of authors without mutual confidence trade between decentralization systems and the throughput a system can handle in a given amount of time. This trade-off should be taken into consideration when determining whether or not to use a blockchain program (Tschorsch and Scheuermann, 2015).

\subsection{Blockchain}

Blockchain in general called "encrypted currency", sometimes as a virtual currency, more specifically as money, and thus perceived by users and subscribers (Tu \& Meredith, 2015). The first definition in the Blockchain portal says that "Blockchain is an innovative payment network and a new type of money" (Institute for the World Economy \& EU, 2018); (Max Kubát, 2015).

\subsubsection{Why we Need Blockchain}

Technology serves purpose in many ways (Coleman, Leary, \& Asmal, 1974)(Brosens, 2017). Most people are not interested in how smartphones or the Internet work, if they benefit from what they do. In addition, this modern digital money is need for modern digital transaction. Below we demonstrate the usages and the necessity of blockchain in our daily life (Yaarub et al., 2018).

\subsubsection{Each began their Own Bank}

The purpose of the cryptocurrency is to become someone's bank (Szczepański, 2014). Basically, this allows an institution to depend on storing the value and sending the planet from one place to another. It seems simple, but the consequences of the mind are incredible (Mandeng, 2018).

\subsubsection{Financial Inclusion}

More than 2 billion people are still bankrupt (Seetharaman et al., 2017). Financial inclusion is key to reducing poverty. If the banks do not want to give a bill to someone because they are considered "cost" or "responsibility", then participation will be very difficult (FSB, 2018). Cryptography allows people to participate in the global economy. Moreover, no frontier, bank or political power can deny the right to save, send and receive money whenever they wish (Seetharaman et al., 2017).

\subsubsection{Fast and Free Transaction}

We do not think we have to explain the enormous benefits of not having to wait three days or even a week for the international transfer (Conti et al., 2018). Not to mention the high fees that banks are making. This is quite novel. Forget that we will get terrible transactions in those dark moneychangers when we are in a foreign country (Rogers, 2014). If people around the world accept crypto currencies, we will never have to worry about having the right amount of money for us (Review \& 2018, 2018).

\subsubsection{A New Initiative where Nobody and Everybody is in Charge}

Nobody and everyone decide what the price for the precise transaction (Yaarub et al., 2018). This is a global agreement between normal people. There is no local war, political conflict or massive corruption that can cause implosion, or nobody can manipulate it (Kelso Hays \& Valek, 2018). Today, however, we are still in a speculative phase where fear or optimism can spread like a fire hazard. This causes a volatile market (Klabbers, 2017). But in the long term, when the cryptocurrency reaches critical acceptance rates and extremely high margins, I think things are becoming clearer. Also, considering, this is mainly the price of Blockchain compared to the traditional currency, which is now unstable. Blockchain and "altcoins", 
like Ethereum or Litecoin, result in different lives (White, 2014). The fact persists; today some people with a lot of money or influence are still manipulating the price. And believe me: many financial institutions and governments around the world do everything possible to destroy the encryption wallets to achieve their goals (Dumitrescu, 2016).

\subsubsection{Safe and Cheaper Merchant}

We want to enter a more honest world. Scammers have no possibility of cryptocurrency, where they can receive, and test and track payments immediately (Dumitrescu, 2016). In a society with almost no cash, we do not want our finances to depend on an uncertain decision or the exhaustion of the services of an organization (Khadka \& Maharjan, 2017).

\subsubsection{Easy Payment Facility}

We have tried to build a very costly payment scheme in our business (FSB, 2018). Solutions such as Blockchain are however essential to accept cryptography on a website or on a store (BIS, 2015).

This is especially an opportunity for entrepreneurs that are still hesitant to start up a business, as they can build a store at any time without a bank account or stock. They accept payments and use the global public free of charge. If the brand seems to have hit our business philosophy, we should follow the steps below and embrace conventional payments (Jonker, 2018). More enjoyable, lower investment and less risk of starting a simple business will lead to more businesses in developing countries in particular. Once again, the page means that scammers can build new companies easily and claim digital money without offering anything (Brosens, 2017).

\subsubsection{Tax Free Transactions}

The potential benefits to consumers with encryption currencies are simple: as some digital currencies, such as blockchain, are not treated as cash, but transition fees to both the consumer and the merchant are useless, both parties' same money.We pay big costs to buy things, says Kim.If we accept crypto coin without a commercial tool, we can still convert the cryptographic payments into cash. Coinbase works mainly to do this and goal is to do cashless crypto currency (Institute for the World Economy \& EU, 2018).

\subsubsection{Digital currencies Find A Foothold in Retail Industries}

Although cryptographers are still speculative tools, most experts agree that the block network will be able to revolutionize trade (Weldon et al., 2017). The cryptographer has a place to grow, but its core value the ability to create layers of trust without a third party (BIS, 2015).

Many startups and crypto evangelists agree that buying cryptocurrencies will be everywhere if we cannot only benefit from decentralized processes (Crospy et al., 2015). But we are in the first days to start we economic role and monetary system in retail market (Seetharaman et al., 2017). Retailers must be sure that it is possible and have time to do so. In addition, blockchain make it easier for merchants and consumers to use digital currencies, retailers can choose different risks according to their appetite (Kakavand et al., 2016).

\subsection{Issues of Blockchain}

The current retail industries are growing very faster and dramatically technology base (Shadab, 2014). The traditional monetary system is very slow, costly and time killing for modern retail system. The retail buys and selling process is very much old and not sufficient for online and crypto payment. In addition, there is no further initiative for customer payment 
policy and initiate crypto payments. This lake of crypto payment is causing an issue for modern retail society (Tu \& Meredith, 2015).

There is little blockchain system in retail industries. They are not really exposed and inactive in real world. However, it slightly reduces the difficulties of retail consumer facility and lightly reduces the overall production cost. In addition, this facility is inactive in diverse operation. Furthermore, it is not effective and not appropriate for real world. It is time to initiate new working management for retail industries for further improvement.

The willingness to accept cryptographic payments like the blockchains used by online retailers is really important for the introduction of blockchains in the retail market. Nakamoto (2008) introduced the world's first decentralized encryption currency, blockchain. Since cryptocurrencies do not meet all the characteristics of money, the term "cryptography" is used in the rest of the document instead of the cryptocurrency (Jonker, 2018). The new Crypto payment means a new payment technology that cannot be paid and can be sent directly to each other electronically and anonymously without having to use the services of trusted third parties, such as financial institutions (FSB, 2018); (Nakamoto, 2008). This allows them to be part of the traditional retail payment market with payment service providers. That is why it is very important to put this new technology in our retail market. (WorldCoinIndex, 2018).

The transaction security factors related to the use of blockchain to blockchains have been introduced in the section and the security cases for blockchains are discussed in the section (BIS, 2015). If the user's data is published in the cloud computing environment, the psychological and financial damage may be caused by the leak of confidential user data. For the most part, data security and data transfer, such as secrecy and integrity, are found in the cloud computing environment. Keep in mind, however, that privacy and anonymity studies are not enough. Blockchain is a representative technology to guarantee anonymity (Rogers, 2014). In addition, currently the combination of the computing environment in the cloud, the block switch is lack of extended to a convenient service that could provide greater security (Boucher et al., 2017).

The blockchain experiment is important to drive this modern crypto payment in retail market (Doroftei, 2016). In order to use this crypto technology, we need proper knowledge and guideline such as if the electronic wallet is not deleted correctly, user information can be omitted. The remaining information of the user can be used to create user information (Shadab, 2014). The main problem is the falsification of the main book or blockchain and the double transaction of the block. Security wallet is required to solve such security problems. Although the electronic wallet that is installed in a computer is usually used, the security of our mobile electronic money should be verified because mobile devices are very popular (Schatsky \& Muraskin, 2015). Because the transaction is based on the time value of a mobile device, the security of the transaction can only be confirmed once the integrity and accuracy of the time stamp created on the mobile device is guaranteed (Park \& Park, 2017).

Consumer demand, net transaction benefits and perceived adoption policies affect the intention to introduce and actual acceptance of retailers Regarding non-financial factors (Jonker, 2018). In addition, our findings suggest that service providers who act as intermediaries among retailers, their customers and payment service providers play a key role in the competition and to facilitate innovation in the online retail payment market by reducing such barriers (Nicole Jonker, 2018). The worst obstacle to cryptographic acceptance seems to be the lack of consumer demand due to its well organization (Jonker, 2018). Informing consumers indicates that those who do not use crypto it for online payment. Therefore, it seems unlikely that the adoption of crypto by retailers will increase significantly, which makes it extremely 
unlikely that crypto like Blockchain will drastically change the existing retail payment system (Nicole Jonker, 2018).

As the current crypto payment system is very complex and the transaction facilitators are scattered, the points affected by the security attacks are increasing (Park \& Park, 2017). In addition, crypto currency can change monetary system as instant a merchant who intends to pay an annual membership fee to receive a card and use it to buy goods or use services (Park \& Park, 2017). The customer's bank and the merchant bank cooperate in the payment of the rate and obtain a card from the bank to use it and use it for the purchase of goods and services. We need to simplify our transaction because more people use smartphones to buy goods or services.

Decentralized cryptographic "currencies", such as blockchain, can drastically change the existing retail payment system and even the monetary system. Therefore, it is crucial to examine the factors that influence its reception (Stevens, 2017). A large cross-section of retailers selling online in their products find that cryptographic payments are currently modest (2\%), but retailers are willing to accept cryptographic payments soon (Schuh \& Shy, 2016).

In addition, contemporary transactions are vulnerable to critical data leakage when the administration server is hacked because all valuable data is handled by the central server. On the other hand, it is very difficult to attack the transactions based on blocks, since all the important data are distributed, and the attacker must hack and change $51 \%$ of the transactions of equal to equal. Therefore, the improved block network must be used for transactions to solve the problems of traditional transactions. One of the biggest problems with using blockchain with the block channel is the possibility of a double transaction. Therefore, the blockchain in retail market needs for further improvement of safe and secure digital transaction instead of contemporary monetary system.

\subsection{Research Question}

In summary, the academic literature provides more information on the factors that influence the decision of retailers to accept payments from a particular customer payment instrument. The literature also suggests that, due to the heterogeneity of retailers, they may think differently about the added value of their business to accept cryptographic payments. Considering this background, this study aims to answer the following key research question:

- Do blockchain experience, Blockchain supervision andBlockchain operation strategy influence on Implementation of Blockchain in Retail Market?

- Do blockchain experience, Blockchain supervision andBlockchain operation strategy effect blockchain adaption.

- Does blockchain adaption affect their Implementation of Blockchain in Retail Market?

- To what extent, does blockchain adaption mediate the relationship between blockchain experience, Blockchain supervision andBlockchain operation strategy?

\subsection{Research Objectives}

Pertaining to this problem statement, this research has addressed the following research objectives:

- To examine the relationship of blockchain experience, Blockchain supervision andBlockchain operation strategy influence toward Implementation of Blockchain in Retail Market?

- To examine the blockchain experience, Blockchain supervision andBlockchain operation strategy on blockchain 
adaption.

- To examine the relationship between the demand necessary blockchain adoption towards Implementation of Blockchain in Retail Market?

- To examine the mediation effects of blockchain adaption towards the relationship between blockchain experience, blockchain management, Blockchain operation strategy with Implementation of Blockchain in Retail Market?

\subsection{Scope of the Study}

This thesis explores blockchain as an investment and examines a blockchain phenomenon of Malaysia. This thesis has four main objectives. This is done by analyzing interviews with the selected stakeholders. This analysis focuses on the general reactions and adoption of blockchains and obstacles. The scope of the study covers the medium retail industries in Malaysia. The researcher examined the limitation and identified the challenges to implement of crypto payment in retail industry Malaysia and management authority to expand the existing retail sector by transforming the Blockchain.

\subsection{Research Significance}

This thesis introduces very new research on retail supply chain using block chain crypto currency. In addition, we argue that the potential cost savings would be significant versus current costs and ongoing maintenance of existing legacy. Blockchain is relatively easier to handle supply chain management and to replicate currency into crypto currency.

\subsubsection{To the Body of Knowledge}

Mainly, the research contributes a new information or knowledge to improve the Retail business initiative through Blockchain that is Blockchain in retail market. It can enhance the retail marketing process and an easy procedure for supply chain management. Clients and retailer have a wonderful dealing and cooperative behavior in local market.

\subsubsection{To the Potential Clients}

Malaysia can be promoted as a distinguishing retail market in local market. Clients can find an easy access in the local market currency using crypto currency that is Blockchain.

\subsubsection{To the Stakeholders}

The blockchain provides an ultimate solution for retail market in Malaysia. The crypto currency highlighting the local and countryside retail business in Malaysia. It will change the business strategies and improve the live hood through blockchain in retail market.

\subsubsection{To the Policy Makers}

The output of this research can help retail industries to promote Malaysian retail industries globally, precisely for the Kuala Lumpur, Kedah and Penang. It can help the government to revive culture in the city and countryside market. The new crypto currency dose not solves only the local retail market, but it can also be used for other business initiatives in the international business. It Blockchain can provide an ultimate management policy for ministry of finance in Malaysia.

\subsection{Research Framework}

Interdependence theory conceptualizes and comprehensively analyzes interpersonal processes and structure (Kelley and 
Thibaut, 1978, Kelley et al. 2003, Rusbult\& Lange, 2003, Thibaut \& Kelley, 1959). Here is a significant emphasis on the relationship between personality (Rusbult\& Van Lange, 2003), which is held by the Implementation of Blockchain in Retail Market (BRMI). Relationships, trust, co-ordination and co-operation are necessary for social interaction, which receives considerable attention (Rusbult\& Van Lange, 2003). Interaction does not necessarily result in specific results, pleasure or dissatisfaction, but direct experience as general symbolic results or experiences (Holmes, 1981, Kelley, 1979). People with relation-specific motivation respond to a specific object in a situation (Rusbault\& Van Lange, 2003), as well as facing people. Brands from this perspective interact with blockchain experience, Blockchain management, and Blockchain operation strategy. In return, they expect the outcomes from blockchain adaption such as commitment, convertibility, trust and passion to continue the blockchain implementation in retail market.

The adaptation of Blockchain is a strong commitment, which is a transactional policy for the administration of Blockchain (Rusbult and Van Lange, 2003). The involvement of the interaction with the partner creates an emotional reaction and improves the habit and long-term participation (for example, Agnew, Van Lange, Rusbult and Langston, 1998). Transformations of Prosocial originate from benevolent thoughts (Rusbult and Van Lange, 2003). Pro-social activities, including forgiveness, accommodation and victims are accentuated by the partners' growing commitment to interaction (eg, Finkel, Rusbul, Kumashiro and Hannon, 2002, Rusbult, Verette, Whitney, Slovik, Lipkus 1991, Van Lange Harinck, Steemers, 1997). Therefore, satisfaction is an important component of the relationship process. To comply with this process, in the framework of the current research, blockchain adaptation has been incorporated between blockchain and the retail market.

To understand the adaptation and interaction of this theory, it is necessary to know the "need" or "dependence" of the pending partners (Rusbult and Van Lange, 2003). The adaptation of Blockchain is related to the interdependence in comfort or avoidance, as a result of dependency, which determines the maintenance or interruption of a relationship. Likewise, addiction is linked to vulnerability because it strengthened unilateral domination and incompatibility. The least controversial partner is to follow the self-interest or the interest of the partner in a situation of non-reciprocal and rather conflicting interests, while the multiple partners recognize the response capacity of the partners. It is considered that the partner is a reliable partner if he responds in a pro-social manner and responds without taking advantage of the power of the addictive position, although the continuity of the relationship may continue in the abusive relationship. This is an important investment or a bad alternative (Johnson, 1995, Rusbult\& Martz, 1995). This positive blockchain experience, the Blockchain supervision and the Blockchain operation strategy foster the adaptation, passion and commitment of blockchain, ultimately driving the blockchain retail market. The theoretical framework is given in figure 2.2. 


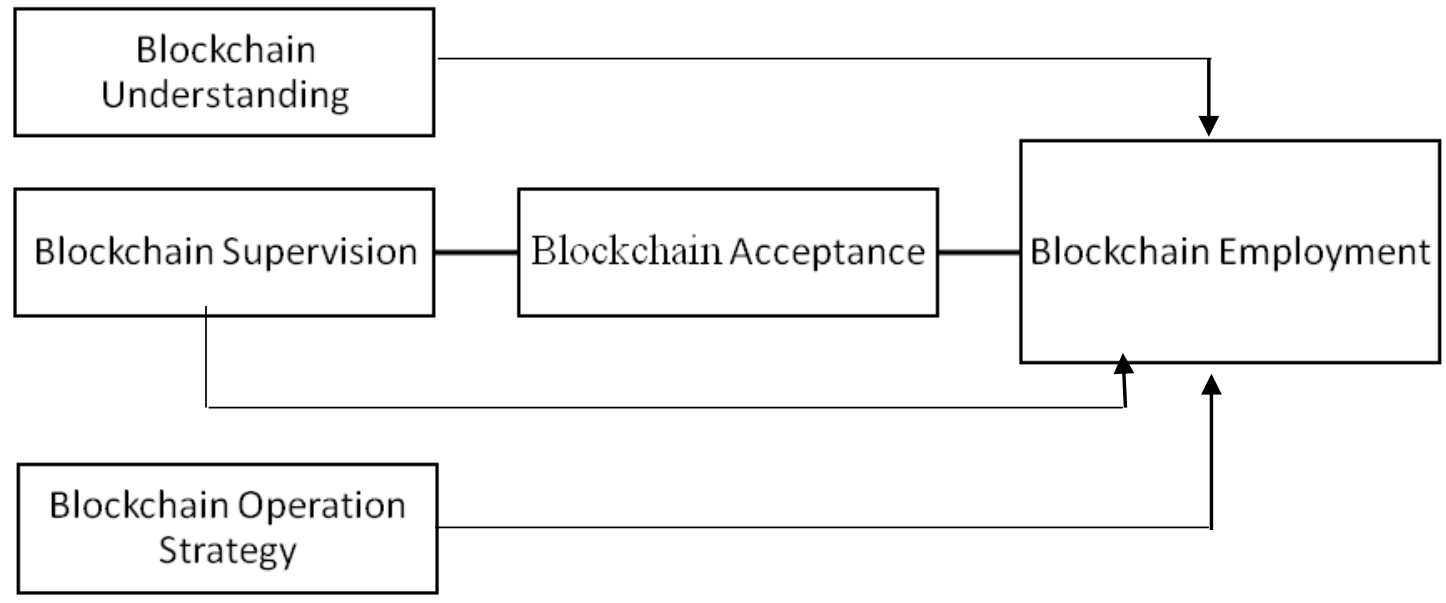

Figure 2.2: Research Framework.

The Framework presented in this article can be described for the theory of the SET theory and the theory of interdependence, because when two people interact, relations develop and maintain through the interdependencies of each (Blau, 1964). However, the researcher preferred the theory of interdependence instead of the theoretical theory of the SET principle for some reasons. The theory of interdependence extends to a wide range of social interactions around the most complete theories (Van Lange and Balliet, 2015). This provides a useful analysis of cognition, emotions, dispositions, norms and interpersonal processes (Rusbult and Van Lange, 2003). The theory comprehensively analyses behavior, cognition and motivation in long-term relationships (Rusbult and Van Lange, 2003). Rusbult and Van Lange (2003) added that the most important force of this theory is the integration of close ties, intergroup behavior and prosocial behavior. In addition, the theory of interdependence covers the foundations and properties of the theory of social exchange. Therefore, the researcher applied the theory of interdependence as underpinning for research.

In addition, the theory of interdependence can be used as a geological theory in business-related research, as Hunt (1995) thinks that Thibaut and Kelley (1978) have studied the paradigm shift in the study of channel conflict. Similarly, Gassenheimer, Calantone and Schully (1996) examined relationships with consumers and retailers. It has been shown that most of the purchases were made by suppliers that participate in wholesale satisfaction. In the same way, from the perspective of the implementation of the blockchain retail market, the theory of mutual dependence of Thibaut and Kelley (1978) is one of the theories of the land. The seminaries of Fournier (1998) are based on the theory of the interdependence of Thibaut and Kelley (1978) and the theory of attraction (Cayolla and Loureiro, 2014, Loureiro, Ruediger, Demetris, 2012). The dimensions of Implementation of Blockchain in Retail Market were also derived from the theory of interdependence between Thibaut and Kelley (1978). In addition, Fritz, Lorenz and Kempe (2014) also use the theory of interdependence to make the blockchain retail market a reality to identify the dimension of the relationship. Algesheimer, Dholakia and Hermann (2004) investigated the theory of the interdependence of the retail community's research, which analyzed the history and consequences of the blockchain of the retail market for members of the automobile club. They argued that the theory of interdependence was useful in the analysis the factor of the Blockchain implementation in retail market. 
In addition, the framework developed in the research based on the theory of autonomy is in line with the Framework of Keller (2001), since this framework can be explained by the brand value Framework of Keller (2001). Blockchain understanding and blockchain transaction are in line with the adaption of the blockchain, Blockchain supervisions in line with the Framework of Blockchain adaption is a factor of blockchain implementation in retail market.

\subsection{Research Hypotheses}

In line with the research objectives presented earlier in the Chapter One and base on the research framework developed in this chapter, the following hypotheses were developed.

\subsubsection{Blockchain understanding and Blockchain Implementation in Retail Market}

Blockchain understanding and cryptocurrency payments like blockchain by online retailers. Nakamoto (2008) introduced the world's first decentralized crypto currency, called blockchain. Cryptos like blockchain represent a new payment technology, which enable payers and payees to directly send value to each other electronically and anonymously without the need to use the services of trusted third parties, like financial institutions (Nakamoto, 2008). This allows them to move outside the scope of the traditional retail payment market with its regulated payment service providers. According to the literature of the bilateral market, the adoption decisions of the retailers depend on the acceptance of the other side of the demand, that is, the blockchain experience. We use several variables that reflect the Blockchain understanding (Weldon et al., 2017). Many articles provide average scores for these variables for respondents who accept crypto and for those who do not. For the last group, the averages are given according to the level of acceptance intention. In addition, we have found that most of the literature shows the positive reality of the Blockchain understanding and blockchain implementation retail market (Szczepański, 2014).

H1A: Blockchain experiences have a significant positive effect on Blockchain Implementation in Retail Market.

\subsubsection{Blockchain Supervision and Blockchain Implementation in Retail Market}

We found that the retailer's assessment of the adoption of Blockchain supervision by the consumer has a significant impact on the blockchain implementation in retail market, and the indicator also influences the blockchain administration (Seetharaman et al., 2017). In the literature, the management of blockchain is positively influenced by the comprehensive evaluation of retailer or consumers of online purchases. The average marginal effects show that a survey of percentage points of cryptographic introduction among consumers increases the probability that retailers want to accept cryptographic payments (Randall et al., 2017).

H1B: Blockchain supervision has a significant positive effect on Blockchain Implementation in Retail Market.

\subsubsection{Blockchain Operation Strategy and Blockchain Implementation in Retail Market}

Several factors reflect the net transactional benefits of a retailer's Blockchain operation strategy, which significantly affect the Blockchain employment and are highly related to the acceptance of cryptography (Moore et al., 2018). Retailers looking for comparatively favorable costs for blockchain transactions compared to other payment instruments are relatively favorable in the implementation of the blockchain retail market. We also see that blockchain's transaction policy, due to relatively fewer working hours than other payment instruments, shows retailers a relatively large willingness to implement the blockchain retail market. At the same time, the estimated effects of the "exchange rate risk crypt" and the "relatively 
favorable cost of labor time costs" are statistically significant, and the efforts required accepting cryptographic payments are also reflected as control (Blundell-Wignall, 2014).

The results of the variables that reflect the net transaction benefits of the cryptographic transaction are largely in line with the implementation of the blockchain retail market. It may be the first time that retailers who manage the security of the Blockchain operation strategy are the safest. An alternative explanation may be the translation of the cause-effect relationship; if retailers accept the cryptographic payment policy, they will find that these payments are in relatively few security problems (Yaarub et al., 2018). As regards the residence of customers, there is no significant impact on the policy of the customers of the euro area in cryptographic transactions, unlike the implementation of the blockchain retail market.

$H_{1 C}$ : Blockchain operation strategy has a significant positive effect on Implementation of Blockchain in Retail Market.

\subsubsection{Blockchain Experiences and Blockchain Adaption}

Blockchain understanding is an important initiative to understand the merits and demerits of blockchain utilization of retail market (Rogers, 2014)(Evans et al., 2008). In addition, the blockchain has its own policy which needs the fundamental knowledge to understand and use in retail market. Furthermore, the blockchain is a digital ledger for online transaction in retail market (Doroftei, 2016). Moreover, the Blockchain understanding can be influencing the blockchain adaption in order to implant the blockchain in retail market (Park \& Park, 2017).

$H_{2 A}$ : Blockchain experiences has a significant positive effect on Blockchain adaption

\subsubsection{Blockchain Supervision and Blockchain Adaption}

Blockchain supervisions a policy of blockchain transaction in various market through online (Wust \& Gervais, 2018). The Blockchain supervisioncan influence the blockchain adaption in numerous aspects (Jacobovitz, 2016)(Matsuno, Shibui, Ochiai, Inoya, \& Takakura, 1987). Blockchain supervision might create a sensation to every consumer form domestic and international aspect (Review \& 2018, 2018). The proper Blockchain supervision can give an impact on blockchain adoption which can empower the blockchain employment(Park \& Park, 2017).

$H_{2 B}$ : Blockchain supervision has a significant positive effect on blockchain adaption

\subsubsection{Blockchain Operation Strategy and Blockchain Adaption}

Blockchain transaction rules and regulation is not clear yet. The clear Blockchain operation strategy will enhance the blockchain adaption (Moore et al., 2018). However, the Blockchain operation strategy needs more clearly to the user, so it can be useable to all stage of customer around the globe (Yaarub et al., 2018).

\section{$\mathrm{H}_{2 \mathrm{C}}$ : Blockchain operation strategy has a significant positive effect on blockchain adaption}

\subsubsection{Blockchain adaption and Implementation of Blockchain in Retail Market}

Blockchain adaption is the gap between blockchain and retail market implementation (Oliver, 1980; Zeithaml, 2000). Service providers make efforts to meet retail market that not only satisfy customers but also invigorate brand equity (Beidenbach, Bengtsson, \&Marell, 2015). Early studies on blockchain and retail market proved that there was direct significant positive relationship between blockchain and retail market (Beidenbach, Bengtsson, \&Marell, 2015). 
H3: Blockchain adaption has a significant positive effect on Implementation of Blockchain in Retail Market.

\subsubsection{Blockchain Adaption as Mediator}

For mediation, according to Baron and Kenny (1986), it is essential to have a significant relationship between the prediction variable and the criterion variable, the predictor variable and the intermediate variable, and the transmission of the variable to the criterion variable. Their mediation criterion assumed that there would be no measurement error and that the criterion variable would not cause a mediator. Preacher and Hayes $(2004,2008)$ criticized the mediation criteria of Baron and Kenny, since these assumptions were routinely violated. It was argued that the significant effect of the predictive variable on the criterion variable is not necessarily necessary for the occurrence of mediation. This was supported by other researchers for example Preacher \& Hayes (2008) suggested that researchers could investigate mediation in a situation where it was possible, theoretically and procedurally, to determine the causal relationship between the predictor, the mediator and the criterion variable. The researcher followed Preacher and Hayes (2008) in seeking mediation and proposed client satisfaction as an intermediary. Customer satisfaction as a mediator is well founded in marketing literature (eg, Caruana, 2002, Chi \&Gursoy, 2009, Garbarino\& Johnson, 1999). Similarly, Park and Lee (2005) stated that the blockchain adaptation link to the Blockchain operation strategy when it was thought that some transaction policy was valuable and useful to them.

From an empirical perspective, satisfaction as a mediator is well founded in the literature. The adaptation of Blockchain as an intermediary played an important role in the implementation of the retail market. Satisfaction played a similar mediating role between the mediator between the adaptation of blockchain and the implementation of the retail market. Similarly, the adaptation of blockchain mediated the connection between the blockchain transaction and the Implementation of Blockchain in Retail Market, while the studies argued that Blockchain supervisionis also the result of the implementation of the retail market of blockchain. Therefore, the researcher has taken the following hypotheses:

H4A: Blockchain adaption mediates the relationship between Blockchain supervisionand Blockchain Implementation in Retail Market.

H4B: Blockchain adaption mediates the relationship between Blockchain understanding and Implementation of Blockchain in Retail Market.

H4C: Blockchain adaption mediates the relationship between Blockchain operation strategy and Implementation of Blockchain in Retail Market.

\subsection{Research Design}

The research design is a fundamental reason for rational decision-making alternatives (Sekaran and Bougie, 2010). This is a research process that is used in this research, and the process extends decisions from general assumptions through methods of data collection and analysis to results (Creswell, 2007). Current research is correlated in nature, since researchers collects data on variables and analyses relationships in the theoretical framework (Cooper and Schindler, 2006). The researcher follows deductive thinking, which is in line with the positivist approach. The quantitative research that followed the investigation followed. The researcher intent to develope hypotheses based on past relevant theory and literature that have been teste using statistical applications. The same study will collecte as cross-sectional data at a given time (Sekaran \& Bougie, 2010) and conclusions will draw from the examination of the given moment. For these data, the 
study collects the individual consumers of various customer of retail market in Malaysia considering the concept of linking the Blockchain experience, the Blockchain management, the policy and implementation of the Blockchain transactional retail market, the intermediary effect of the Blockchain adaptation.

In this document, a quantitative research method uses, and the main research tool consists of a structured questionnaire. The survey will be conducted using the structure questionnaire. The social science study structure questionnaire is use to study variables such as opinions, preferences, beliefs and attitudes, and the survey method is the best social gathering and attitudes, facts and beliefs of the staff (Kerlinger, 1973). Finally, a statistical analysis will performed to obtain empirical support for the probability relation between the variables.

\subsection{Data Analysis}

In this section this study describes data analysis and result

\subsubsection{Data Analysis Tools}

We used a pilot study of the Social Science Statistical System (SPSS v23). The Smart PLS (Small Test Squire) 3.0 is also used, since the goal is to predict a complicated structures model. That is why we used PLS. Data are not usually distributed (Miraz et.al, 2020).

\subsubsection{Population of the Study}

People, events or objects are included in the population and we draw conclusions from these results. A retailer in Malaysia is the focus community for this analysis. The sample will be obtained from both dealer, operator and distributor customers. Therefore, this study chooses customer and retail management (Hair et.al, 2017).

\subsubsection{Sampling Technique and Data Collection}

Systematic sampling is a type of probabilistic samples. In systematic sampling, when the first element is randomly picked from the population, the elements are systematically or at certain intervals. Yet Malhotra (2014) said that systematic sampling the be used for the collection of data from the final respondents in which the complex shopping survey structure for the target population is used.

\subsubsection{Result}

Fornell-Larker criterion of Discriminant Validity shows in below.

Table 1: Fornell-Larker Criterion

\begin{tabular}{|l|c|c|c|c|c|}
\hline & BS & BU & BOS & BA & BE \\
\hline BS & 0.82 & & & & \\
\hline BU & 0.44 & 0.643 & & & \\
\hline BOS & 0.474 & 0.316 & 0.489 & & \\
\hline BA & 0.243 & 0.465 & 0.297 & 1.00 & \\
\hline BE & 0.412 & 0.654 & 0.544 & 0.576 & 1.00 \\
\hline
\end{tabular}

HTMT Ration of Discriminant Validity describe below. 
Table 2: HTMT Ration

\begin{tabular}{|l|c|c|c|c|c|}
\hline & BS & BU & BOS & BA & BE \\
\hline BS & & & & & \\
\hline BU & 0.399 & & & & \\
\hline BOS & 0.801 & 0.423 & & & \\
\hline BA & 0.301 & 0.398 & 0.342 & & \\
\hline BE & 0.543 & 0.732 & 0.489 & 0.564 & \\
\hline
\end{tabular}

Variance of Inflated Factor (VIF) show in this below.

Table 3: Variance of Inflated Factor

\begin{tabular}{|l|c|c|}
\hline & BA (Blockchain Acceptance) & Be (Blockchain Employment) \\
\hline Bs (Blockchain Supervision) & 1.182 & 1.325 \\
\hline Bu (Blockchain Understanding) & 1.483 & 1.113 \\
\hline Bos (Blockchain operation strategy) & 1.624 & 1.132 \\
\hline Be (Blockchain Employment) & 2.342 & \\
\hline
\end{tabular}

\subsection{DISCUSSIONS}

Finally, Blockchain is the backbone of Blockchain technology. The shared accounting functions offer a very attractive technology to protect current financial and non-financial business problems along with the security of blockchain. When it comes to technology, the technology based on cryptocurrencies or inflated expectations are very depressed.

\subsection{SUMMARY}

Certain types of block chains were made and developed for various purposes. Despite the development of cohort systems, the block chain needs more development from the perspective of Malaysia. In addition, MyBlockchain is the only cryptographic block currency that improves retail marketing.

This chapter has critically analyzed the variables of the current research Framework of the CBR. The brand experience of variables, brand personality, CRM, customer satisfaction and definitions of CBR, dimensions and implications were discussed gradually. At the end of this chapter, the underlying theory, the theoretical framework and the hypotheses were discussed in detail.

\section{REFERENCES}

1. Allen, M. A. (2018). The Influence of Regional Power Distributions on Interdependence. Journal of Conflict Resolution, 62(5), 1072-1099. https://doi.org/10.1177/0022002716669809

2. Amiot, C. E., Vallerand, R. J., \& Blanchard, C. M. (2006). Passion and psychological adjustment: A test of the personenvironment fit hypothesis. Personality and Social Psychology Bulletin, 32(2), 220-229. https://doi.org/10.1177/0146167205280250

3. Bäckström, K., \& Johansson, U. (2006). Creating and consuming experiences in retail store environments: Comparing retailer and consumer perspectives. Journal of Retailing and Consumer Services, 13(6), 417-430. https://doi.org/10.1016/j.jretconser.2006.02.005

4. Sinsupa Wannasuth, "The Increase in Synergy's Value of Supply Chain's Triangle Corners for Retail Business ", BEST: International Journal of Management, Information Technology and Engineering (BEST: IJMITE), Vol. 3, Issue 12, pp. 1-6 
5. Balliet, D., Tybur, J. M., \& Van Lange, P. A. M. (2017). Functional Interdependence Theory: An Evolutionary Account of Social Situations. Personality and Social Psychology Review, 21(4), 361-388. https://doi.org/10.1177/1088868316657965

6. Bansal, S., \& Bansal, I. (2018). Consumer engagement in electronic word-of-mouth on social networking sites. ELK Asia Pacific Journal of Marketing and Retail Management, 7(2), 1-27. https://doi.org/10.16962/EAPJMRM/issn.

7. BIS. (2015). Committee on Payments and Market Infrastructures Digital Currencies. Bank for International Settlements, (November). Retrieved from https://www.bis.org/cpmi/publ/d137.pdf

8. Blundell-Wignall, A. (2014). The Blockchain Question - Currency versus trust-less transfer technology. OECD Working Papers on Finance, Insurance and Private Pensions No. 37, 37(37), 22. https://doi.org/10.1787/5jz2pwjd9t20-en

9. Boucher, P., Nascimento, S., \& Kritikos, M. (2017). How Blockchain Technology Could Change Our Lives. European Parliament, 4-25. https://doi.org/10.2861/926645

10. Braver, T. S., Krug, M. K., Chiew, K. S., Kool, W., Andrew Westbrook, J., Clement, N. J., ... Somerville, L. H. (2014). Mechanisms of motivation-cognition interaction: Challenges and opportunities. Cognitive, Affective and Behavioral Neuroscience, 14(2), 443-472. https://doi.org/10.3758/s13415-014-0300-0

11. Brosens, T. (2017). Why Blockchain is destined to become a niche asset. Ing, (December), 1-8. Retrieved from https://think.ing.com/uploads/reports/171218_Why_Blockchain_to_become_niche_asset__Teunis_Brosens.pdf

12. Coleman, A. J., Leary, W. F., \& Asmal, A. C. (1974). Isoproterenol blockade in man: comparison between penbutalol and acebutolol. Current Therapeutic Research - Clinical and Experimental, 16(1), 64-79.

13. Collection, A. T. C. (1984). Media Handbook. Rockville, MD.

14. Conti, M., E, S. K., Lal, C., \& Ruj, S. (2018). A Survey on Security and Privacy Issues of Blockchain. IEEE Communications Surveys and Tutorials. https://doi.org/10.1109/COMST.2018.2842460

15. Crospy, M., Nachiappan, \& Pradhan, P. (2015). Blockchain Technology Explained. Blockchain Technologies, 1-27. https://doi.org/10.15358/0935-0381-2015-4-5-222

16. Cross, E. J., Overall, N. C., Low, R. S. T., \& McNulty, J. K. (2018). An interdependence account of sexism and power: Men's hostile sexism, biased perceptions of low power, and relationship aggression. Journal of Personality and Social Psychology. https://doi.org/10.1037/pspi0000167

17. D'Alfonso, A., Langer, P., \& Vandelis, Z. (2016). The Future of Cryptocurrency. International Security, 7(5), 7-45. https://doi.org/10.1111/j.1461-0248.2010.01520.x

18. Demertzis, M., \& Wolff, G. B. (2018). The economic potential and risks of crypto assets: is a regulatory framework needed?, (September), 1-14.

19. Donegan, S. L. (2016). Philanthropy vs. Racism: Inside Kellogg's Quest for \&quot;Healing and Transformation\&quot; Inside Philanthropy, 1-35. Retrieved from https://www.insidephilanthropy.com/home/2017/7/24/kellogg-foundation-racialhealing-grants

20. Doroftei, C. (2016). Formaldehyde sensitive Zn-doped LPFO thin films obtained by rf sputtering. Sensors and Actuators, B: Chemical, 231(42), 793-799. https://doi.org/10.1016/j.snb.2016.03.104

21. Dorri, A., Kanhere, S. S., \& Jurdak, R. (2016). Blockchain in internet of things: Challenges and Solutions. https://doi.org/10.1145/2976749.2976756 
22. Dumitrescu, G. C. (2016). Blockchain - A Brief Analysis of the Advantages and Disadvantages. Global Economic Observer, 5(2), 63-71.

23. Evans, J., Bridson, K., Byrom, J., \& Medway, D. (2008). Revisiting retail internationalisation: Drivers, impediments and business strategy. International Journal of Retail and Distribution Management, 36(4), $260-280$. https://doi.org/10.1108/09590550810862679

24. Free, L., Macarthur, J. R., Becker, G., \& Laureate, N. (n.d.). Blockchain and the Future.

25. FRY, J. E. A. \& B. (2016). This Version Does Not Contain Page Numbers Article Is Blockchain a Security?

26. FSB. (2018). Crypto-asset markets - Potential channels for future financial stability implications, (October).

27. Ganji Jamehshooran, B., Shaharoun, A. M., \& Haron, H. N. (2015). Assessing Supply Chain Performance through Applying the SCOR Model. International Journal of Supply Chain Management, 4(1), 1-11.

28. Garcia-Santillan, A., Moreno-Garcia, E., Carlos-Castro, J., Zamudio-Abdala, J. H., \& Garduno-Trejo, J. (2012). Cognitive, Affective and Behavioral Components That Explain Attitude toward Statistics. Journal of Mathematics Research, 4(5). https://doi.org/10.5539/jmr.v4n5p8

29. Geisler, M. (2014). The Impact of the Game Theory in Supply Chain Management, 1(3), 11-18.

30. Gregory, J. L., \& Noto, L. A. (2018). Attitudinal instrument development: Assessing cognitive, affective, and behavioral domains of teacher attitudes toward teaching all students. Cogent Education, 4(1), 1-12. https://doi.org/10.1080/2331186X.2017.1422679

31. Hair Jr, J. F., Hult, G. T. M., Ringle, C., \& Sarstedt, M. (2017). A primer on partial least squares structural equation modeling (PLS-SEM). California: Sage Publications.

32. Hertzog, D., \& Ramsey-Musolf, M. J. (2012). Parity- and Time-Reversal Tests in Nuclear Physics. https://doi.org/10.1142/9789814425810_0006

33. Hoang, A. (2017). Blockchain: the Added Values and Challenges of Adopting Blockchain . Bachelor's Thesis, Turku University of Applied Sciences, 65 pages.

34. Hobson, D. (2013). What is Blockchain? XRDS: Crossroads, The ACM Magazine for Students, $20(1), 40$. https://doi.org/10.1145/2510124

35. Huhtinen, T.-P. (2014). Blockchain as a monetary system: Examining attention and attendance. Retrieved from http://epub.lib.aalto.fi/en/ethesis/pdf/13626/hse_ethesis_13626.pdf

36. Institut, W. I. G. (2017). Blockchain : Blockchain :

37. G. RAJESH \& S. NARAYANA RAJAN2, " An Assessment of Service Quality of Organized Super Markets in Kerala ", International Journal of Business and General Management (IJBGM), Vol. 6, Issue 6, pp. 57-66

38. Institute for the World Economy, K., \& EU. (2018). QVirtual Currencies, (July).

39. International, A., \& Access, O. (2016). An Overview of Information Technology Tools Implementation in Supply Chain Management Mahadi Hasan Miraz 1, 2(2), 110-117.

40. Jacobovitz, O. (2016). Blockchain for Identity Management. Technical Report Ben-Gurion University, (December), 1-19. Retrieved from https://www.cs.bgu.ac.il/ frankel/TechnicalReports/2016/16-02.pdf 
41. Jahani, E., Krafft, P. M., Suhara, Y., Moro, E., \& Pentland, A. (2018). ScamCoins, S*** Posters, and the Search for the Next Blockchain $^{T M}$ : Collective Sensemaking in Cryptocurrency Discussions. Proceedings of the ACM on Human-Computer Interaction, 2(November), 79. https://doi.org/10.1145/3274348

42. Jardine, E., Morrison, M., \& Morrison, R. M. (2018). An Analysis : Complex Interdependence and the Chinese-United States Cyber Relationship Robert Maxwell Morrison Thesis submitted to the faculty of the Virginia Polytechnic Institute and State University in partial fulfillment of the requirements for the de.

43. Jonker, N. (2018). What Drives Blockchain Adoption by Retailers. Ssrn, (585). https://doi.org/10.2139/ssrn.3134404

44. Kakavand, H., Kost De Sevres, N., \& Chilton, B. (2016). The Blockchain Revolution: An Analysis of Regulation and Technology Related to Distributed Ledger Technologies. Ssrn. https://doi.org/10.2139/ssrn.2849251

45. Kelso Hays, D., \& Valek, M. J. (2018). crypto research report June 2018, (June), 66. Retrieved from https://derinet.vontobel.com/CH/Download/AssetStore/e99049b4-2393-4bad-b6c5-65ee788c1e26/Crypto-Research-Report-IIDeutsch.pdf

46. Khadka, K., \& Maharjan, S. (2017). Customer Satisfaction and Customer Loyalty, (November), 56+6. https://doi.org/10.1063/1.1659283

47. Khan, S. A. M. M. H., Habib, M., Nazri, E. M., \& Miraz, M. H. (n.d.). ICT Application in Supply Chain o n Numerous Grounds ' in Education and Industries Assimilation.

48. Khasu, D. S., \& Williams, T. O. (2016). The Score Reliability of Draw-a-Person Intellectual Ability Test (DAP: IQ) for Rural Malawi Students. Journal of International Education Research, 12(2), 61-66. Retrieved from https://search.proquest.com/docview/1826533717?accountid=13042

49. Klabbers, S. (2017). Blockchain as an investment asset: The added value of blockchain in a global market portfolio Blockchain as an investment asset, 5-6.

50. Leonard, R. C., Knott, L. E., Lee, E. B., Singh, S., Smith, A. H., Kanter, J., ... Wetterneck, C. T. (2014). The Development of the Functional Analytic Psychotherapy Intimacy Scale. Psychological Record, 64(4), 647-657. https://doi.org/10.1007/s40732014-0089-9

51. Lerner, S. D. (2015). RSK White paper. Retrieved from http://www.the-blockchain.com/docs/Rootstock-WhitePaperOverview.pdf

52. Li, G., Hou, S., Li, Y., Liu, S., Teng, D., \& Hou, D. (2015). Surveillance of gram-positive cocci infections and drug resistance. Cellular and Molecular Biology, 61(4), 90-93. https://doi.org/10.14715/cmb/2015.61.4.15

53. Lo, S., \& Wang, J. C. (2014). Blockchain as Money? Motivation, (14), 1-28.

54. Mandeng, O. J. (2018). Cryptocurrencies, Monetary Stability and Regulation: Century, S Nineteenth Banks, Private Issue, O F. https://doi.org/10.13140/RG.2.2.32541.41448.

55. Malhotra, N. K. (2014). Marketing research: An applied orientation. New Delhi: Dorling Kindersley Pvt. Ltd.

56. Matsuno, A., Shibui, S., Ochiai, C., Inoya, H., \& Takakura, K. (1987). Primary intracranial epidermoid carcinoma accompanied with epidermoid cyst in the cerebellopontine angle - A case report. Neurological Surgery, 15(8), 851-858. https://doi.org/10.1186/s40854-016-0049-2

57. G. Gabriel Prabhu \& G. Duraimurugan, " Growth of FDI in Retailing-A View ", BEST: International Journal of Humanities, 
Arts, Medicine and Sciences (BEST: IJHAMS), Vol. 2, Issue 9, pp. 9-14

58. METI. (2016). Survey on Blockchain Technologies and Related Services FY2015 Report. Nomura Research Institute, (March). Retrieved from http://www.meti.go.jp/english/press/2016/pdf/0531_01f.pdf

59. Miraz, M. H., Ramli, R., \& Mahamud, K.-R. K. (2017). Collaborative web recommender framework for homestay programs. Journal of Engineering and Applied Sciences, 12(6). https://doi.org/10.3923/jeasci.2017.1575.1581

60. Miraz, M. H., Saleheen, F., Habib, M., \& Ramli, R. (2012). IT Operations in Retail Banking, 1-5.

61. Miraz, M., Habib, M., \& Saleheen, F. (2016). ICT-Based Business Initiatives for Women: An Outline of Best Practices in ECommerce/E-Retailing Ventures, 1(1),31-36. https://doi.org/10.22606/fmr.2017.11005

62. Miraz, M. J., Saleheen, F., \& Rahman, M. (2016). Supply chain management in service quality. In Proceedings of the International Conference on Industrial Engineering and Operations Management (Vol. 8-10 March).

63. Moore, T., Christin, N., \& Szurdi, J. (2018). Revisiting the Risks of Blockchain Currency Exchange Closure. ACM Transactions on Internet Technology, 18(4), 1-18. https://doi.org/10.1145/3155808

64. Ndaaba, N. M. M., Harada, Y., Romle, A. R., \& Shamsudin, A. S. (2016). Cognitive, Affective and Conative Loyalty in Higher Education Marketing: Proposed Model for Emerging Destinations. International Review of Management and Marketing, 6(54), 168-175.

65. Neramballi, A., Sequeira, M., Rydell, M., Vestin, A., \& Ibarra, M. (2017). A Comprehensive Literature Review of Green Supply Chain Management, 1-8. https://doi.org/10.11159/icesdp17.176

66. Oliver, P., Marwell, G., \& Teixeira, R. (1985). A Theory of the Critical Mass. I. Interdependence, Group Heterogeneity, and the Production of Collective Action. American Journal of Sociology, 91(3), 522-556. https://doi.org/10.1086/228313

67. Park, J., \& Park, J. (2017). Blockchain Security in Cloud Computing: Use Cases, Challenges, and Solutions. Symmetry, 9(8), 164. https://doi.org/10.3390/sym9080164

68. Personal, M., Archive, R., \& Sovbetov, Y. (2018). M P RA Factors Influencing Cryptocurrency Prices: Evidence from Blockchain, Ethereum, Dash, Litcoin, and Monero. Journal of Economics and Financial Analysis, 2(2), 1-27. https://doi.org/10.1991/jefa.v2i2.a16

69. Pieters, G., \& Vivanco, S. (2016). Federal Reserve Bank of Dallas Globalization and Monetary Policy Institute Financial Regulations and Price Inconsistencies across Blockchain representing $26 \%$ of global blockchain trade volume. These differences must - due to the, (293), 1-45.

70. Pisa, M., \& Juden, M. (2017). Blockchain and Economic Development, (July). Retrieved from https://www.cgdev.org/sites/default/files/blockchain-and-economic-development-hype-vs-reality_0.pdf

71. Queirós, A., Fernandes, E., Reniers, R., Sampaio, A., Coutinho, J., \& Seara-Cardoso, A. (2018). Psychometric properties of the questionnaire of cognitive and affective empathy in a Portuguese sample. PLoS ONE, 13(6), 1-14. https://doi.org/10.1371/journal.pone.0197755

72. Randall, D., Goel, P., \& Abujamra, R. (2017). Blockchain Applications and Use Cases in Health Information Technology. Journal of Health \& Medical Informatics, 08(03), 8-11. https://doi.org/10.4172/2157-7420.1000276

73. Reid, R. C., Li, D. S., Gilliland, R., Stein, J. A., \& Fong, T. (2011). Reliability, validity, and psychometric development of the pornography consumption inventory in a sample of hypersexual men. Journal of Sex and Marital Therapy, 37(5), 359-385. https://doi.org/10.1080/0092623X.2011.607047 
74. Review, S. E.-L. L., \& 2018, undefined. (2018). Blockchain: The First Self-Regulating Currency? Eprints.Lse.Ac.Uk. Retrieved from http://eprints.lse.ac.uk/88095/

75. Rhodes, C. (2018). Retail sector in the UK, (October), 1-15.

76. Rogers, E. M. (2014). The Adoption Process of cryptocurrencies. Journal of Cooperative Extension, 16-22. Retrieved from https://pdfs.semanticscholar.org/5c0d/bbf5c9aa38766d61eac90a0258b4d7d97f6f.pdf

77. Rusbult, C. E., \& Van Lange, P. A. M. (2003). Interdependence, Interaction, and Relationships. Annual Review of Psychology, 54(1), 351-375. https://doi.org/10.1146/annurev.psych.54.101601.145059

78. Saleheen, F., Miraz, M. H., Habib, M. M., \& Hanafi, Z. (2014). Challenges of warehouse operations: A case study in retail supermarket. International Journal of Supply Chain Management, 3(4).

79. Santhana, P. (2015). Blockchain and Analytics Assessing the opportunities and vulnerabilities of the cryptocurrency marketplace. Deloitte.

80. Schatsky, D., \& Muraskin, C. (2015). Beyond blockchain: Blockchain is coming to disrupt your industry. Deloitte University Press, 1-27.

81. Schuh, S. (2015). U. S. Consumers' Adoption and Use of Blockchain and other Virtual Currencies *.

82. Seetharaman, A., Saravanan, A. S., Patwa, N., \& Mehta, J. (2017). Impact of Blockchain as a World Currency. Accounting and Finance Research, 6(2), 230. https://doi.org/10.5430/afr.v6n2p230

83. Shadab, H. B. (2014). Regulating Blockchain and Block Chain Derivatives. Ssrn, 1-22. https://doi.org/10.2139/ssrn.2508707

84. South African Reserve Bank. (2014). Quarterly Bulletin 2014. Quarterly Bulletin, 54(3).

85. Szczepański, M. (2014). Blockchain: market, economics and regulation. European Parliamentary Research Service, 1-9.

86. Taylor, J. B. (2001). a N a Nalysis of the in. Public Policy, (June), 107-132.

87. Trust, W., \& Advisors, I. (2017). The ABCs of blockchain And a look at its investment potential, 1-16.

88. Tsanidis, C., Nerantzaki, D.-M., Karavasilis, G., Vrana, V., \& Paschaloudis, D. (2015). Greek consumers and the use of Blockchain. The Business \& Management Review, 6(2), 295-302. Retrieved from http://www.abrmr.com/myfile/conference_proceedings/Con_Pro_52588/2015icbed35.pdf

89. Tschorsch, F., \& Scheuermann, B. (2016). Blockchain and beyond: A technical survey on decentralized digital currencies. IEEE Communications Surveys and Tutorials, 18(3), 2084-2123. https://doi.org/10.1109/COMST.2016.2535718

90. Tu, K. V, \& Meredith, M. W. (2015). Rethinking Virtual Currency Regulation in the Blockchain Age. Washington Law Review, 90, 271-347. https://doi.org/10.1525/sp.2007.54.1.23.

91. Villalobos, C., Caballero, E., Sanz-Blasco, S., \& Núñez, L. (2012). Study of neurotoxic intracellular calcium signalling triggered by Amyloids. Methods in Molecular Biology, 849(October), 289-302. https://doi.org/10.1007/978-1-61779-5510_20

92. Weldon, R., Herridge, M., Cohen, J., \& Technology Solutions, C. (2017). Retail: Opening the Doors to Blockchain, (July), 128. Retrieved from https://www.cognizant.com/whitepapers/retail-opening-the-doors-to-blockchain-codex2879.pdf

93. Gaurav Tyagi," Relationship between Customer Satisfaction and Price: A Research on Retailers in Bangalore City ", International Journal of Business and General Management (IJBGM), Vol. 3, Issue 1, pp. 31-38 
94. White, L. H. (2014). The Market for Cryptocurrencies. Ssrn, 383-402. https://doi.org/10.2139/ssrn.2538290

95. Wust, K., \& Gervais, A. (2018). Do you Need a Blockchain? 2018 Crypto Valley Conference on Blockchain Technology (CVCBT), 45-54. https://doi.org/10.1109/CVCBT.2018.00011

96. Yaarub, N. A. H., Alshrefi, S. M., Rafea, F. A. M., Ahmed, T., Al-Mamoori, M. H. K., Al-Nafiey, A. K., \& Al-Kaim, A. F. (2018). Optical and morphological characterizations of element Oxides Compound (Y2O3/SeO2) nano-composite structures synthesized via Chemistry of Laser Ablation. Journal of Global Pharma Technology, 10(3), 536-544. https://doi.org/10.1525/sp.2007.54.1.23.

97. Yli-Huumo, J., Ko, D., Choi, S., Park, S., \& Smolander, K. (2016). Where Is Current Research on Blockchain Technology?-A Systematic Review. PloS One, 11(10), e0163477. https://doi.org/10.1371/journal.pone.0163477

98. Zheng, B. (2017). Blockchain and Blockchain Technology - A Primer, 1-6.

\section{AUTHORS PROFILE}

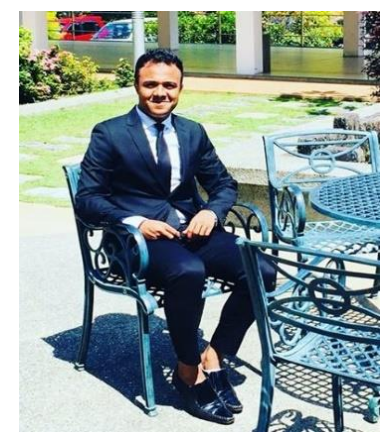

Hello, this is Mahadi Hasan Miraz completed BSC, MSC from Universiti Utara Malaysia (UUM) and a PhD fellow at UUM. Well, I have outstanding Technical, Entrepreneur and leadership skill. Besides, I have involved with Universiti Utara Malaysia numerous FRGS and Government PROJECT, Malaysia. Furthermore, I have published 42+ articles on Management, information technology, Entrepreneurship and supply chain in SCOPUS \& ISI index journal. My core research on Blockchain, IT, Management \& Supply chain. Apart from that, I have vast knowledge about research and analysis Using SEM-PLS, AMOS and SPSS. Also, an expert on JAVA, MS Office, Joomla and Java Script.

Mr. Mohammad Tariq Hasan, he has completed his BBA and MBA from Faculty of Business Studies of Dhaka University in 2005 and 2006 respectively. After completion his graduation, he has started his career in corporate sector as Financial analyst in CRISL, a credit rating company and was part of different company in various position over the period of 2006-09. In 2009, he has joined in ASA University Bangladesh, as a lecturer of Accounting and from that time, he is a part of the education industry and working in different top ranked private university of Bangladesh. At present he is working as Assistant Profess, Accounting in United International University, Bangladesh and doing his PhD from School of Accountancy, Universiti Utara Malaysia. Till now, he has published 20+ (plus) research papers in different National and International peer reviewed journal. 